\title{
Charm and beauty at the LHC
}

\author{
Andrea Dainese \\ INFN - Laboratori Nazionali di Legnaro, \\ viale dell'Università 2, 35020, Legnaro (Padova), Italy
}

\begin{abstract}
The Large Hadron Collider at CERN will open a new energy domain for heavyion physics. Besides ALICE, the dedicated heavy-ion experiment, also ATLAS and CMS are preparing rich physics programs with nucleus-nucleus collisions. Here we focus on open heavy-flavour and quarkonia studies, among the fields that will most benefit from the high centre-of-mass energy at the LHC. We discuss a few examples of physics issues that can be addressed and we present a selection and comparison (where possible) of results on the expected capability of the three experiments.
\end{abstract}

Key words: Large Hadron Collider, heavy-ion collisions, heavy-flavour production PACS: 25.75.-q, 14.65.Dw, 13.25.Ft

\section{Introduction}

The nucleon-nucleon c.m.s. energy for $\mathrm{Pb}-\mathrm{Pb}$ collisions at the $\mathrm{LHC}, \sqrt{s_{\mathrm{NN}}}=$ $5.5 \mathrm{TeV}$, will exceed that available at RHIC by a factor about 30, opening up a new domain for the study of strongly-interacting matter in conditions of high energy density (QCD medium). High initial temperature is expected to characterize the system; the predicted values are on the order of $600 \mathrm{MeV}$, twice as large as at RHIC and four times larger than the critical temperature, $T_{\mathrm{c}} \approx 170 \mathrm{MeV}$ at RHIC and LHC conditions, at which lattice QCD calculations predict the phase transition to a deconfined state of matter [1].

At the LHC, hard-scattering processes should contribute significantly to the total cross section. The mechanism of energy loss due to medium-induced gluon radiation allows to use the energetic partons produced in initial hardscattering processes as probes to collect information on the opacity and density of the medium itself. The set of available probes will be extended both

Email address: andrea.dainese@lnl.infn.it (Andrea Dainese). 
quantitatively and qualitatively. In fact, hard (light)quarks and gluons will be produced with high rates up to very large transverse momentum $\left(p_{\mathrm{t}}\right)$. Additionally, charm and beauty quarks, which, due to their masses, would show different attenuation patterns [2] (section 2), will become available for detailed measurements, since their production cross sections are expected to increase by factors 10 and 100, respectively, from RHIC to the LHC. The entire spectrum of charmonia and bottomonia will be abundantly produced and a comparative measurement of their yields is expected to provide insight on the properties, in particular on the temperature, of the deconfined medium (section 2).

\section{Heavy-quark and quarkonia phenomenology at the LHC}

Heavy-quark pairs, $\mathrm{Q} \overline{\mathrm{Q}}$, are produced in hard scattering processes with large momentum transfer, $Q^{2} \gtrsim\left(2 m_{\mathrm{Q}}\right)^{2}$. Hence, the production cross sections in nucleon-nucleon (NN) collisions can be calculated in the framework of perturbative QCD (pQCD). The expected yields in pp collisions at $\sqrt{s}=14 \mathrm{TeV}$ are reported in the first line of Table 1 [3], as obtained at next-to-leading order using the MNR program [4] with reasonable parameter values (quark masses and perturbative scales) [3]. These yields have large uncertainties, of about a factor 2, estimated by varying the parameters [3].

For hard processes, in the absence of nuclear and medium effects, a nucleusnucleus collision would behave as a superposition of independent NN collisions. The charm and beauty yields would then scale from pp to AA proportionally to the number $N_{\text {coll }}$ of inelastic binary NN collisions. Binary scaling is, indeed, expected to break down due to initial-state effects, such as nuclear shadowing of the parton distribution functions (gluon recombination in the high-density small- $x$ regime), and final-state effects, such as parton energy loss in the medium formed in AA collisions.

In Table 1 we report the $c \bar{c}$ and $b \bar{b}$ yields in $\mathrm{p}-\mathrm{Pb}$ and $\mathrm{Pb}-\mathrm{Pb}$ collisions, obtained including in the NLO pQCD calculation the EKS98 parameterization [5] of the PDFs nuclear modification and applying binary scaling [3]. The charm (beauty) cross-section reduction induced by shadowing is about $35 \%$

Table 1

Expected Q $\bar{Q}$ yields at the $\mathrm{LHC}$, from NLO pQCD [3]. For $\mathrm{p}-\mathrm{Pb}$ and $\mathrm{Pb}-\mathrm{Pb}, \mathrm{PDF}$ shadowing is included and binary scaling is applied.

\begin{tabular}{ccccc}
\hline colliding system & $\sqrt{s_{\mathrm{NN}}}$ & centrality & $N^{\mathrm{c} \overline{\mathrm{c}}} /$ event & $N^{\mathrm{b} \overline{\mathrm{b}}} /$ event \\
\hline $\mathrm{pp}$ & $14 \mathrm{TeV}$ & minimum bias & 0.16 & 0.0072 \\
$\mathrm{p}-\mathrm{Pb}$ & $8.8 \mathrm{TeV}$ & minimum bias & 0.78 & 0.029 \\
$\mathrm{~Pb}-\mathrm{Pb}$ & $5.5 \mathrm{TeV}$ & central $\left(0-5 \% \sigma^{\text {tot }}\right)$ & 115 & 4.6 \\
\hline
\end{tabular}


$(20 \%)$ in $\mathrm{Pb}-\mathrm{Pb}$ and $15 \%(10 \%)$ in $\mathrm{p}-\mathrm{Pb}$. There is a significant uncertainty on the strength of shadowing in the small- $x$ region and some models predict much larger suppression than EKS98 (see [6] for a review). The comparison of $\mathrm{Q} \overline{\mathrm{Q}}$ production in $\mathrm{pp}$ and $\mathrm{p}-\mathrm{Pb}$ collisions is regarded as a sensitive tool to probe nuclear PDFs. In particular, the ratio of invariant-mass spectra of dileptons from heavy-quark decays for the two systems would measure the PDFs nuclear modification [6].

Experiments at RHIC have shown that the nuclear modification factor of particles $p_{\mathrm{t}}$ distributions, $R_{\mathrm{AA}}\left(p_{\mathrm{t}}, \eta\right)=\frac{1}{\left\langle N_{\mathrm{coll}}\right\rangle} \cdot \frac{\mathrm{d}^{2} N_{\mathrm{AA}} / \mathrm{d} p_{\mathrm{t}} \mathrm{d} \eta}{\mathrm{d}^{2} N_{\mathrm{pp}} / \mathrm{d} p_{\mathrm{t}} \mathrm{d} \eta}$, is an effective observable for the study of the interaction of the hard partons with the medium produced in nucleus-nucleus collisions. Heavy-quark medium-induced quenching is one of the most captivating topics to be addressed in $\mathrm{Pb}-\mathrm{Pb}$ collisions at the LHC. Due to the QCD nature of parton energy loss, quarks are predicted to lose less energy than gluons (that have a higher colour charge) and, in addition, the 'dead-cone effect' is expected to reduce the energy loss of massive quarks $[7,8]$. Therefore, one should observe a pattern of gradually decreasing $R_{\mathrm{AA}}$ suppression when going from gluon-originated light-flavour hadrons $\left(h^{ \pm}\right.$or $\left.\pi^{0}\right)$ to $\mathrm{D}$ and to $\mathrm{B}$ mesons [2,9]: $R_{\mathrm{AA}}^{h} \lesssim R_{\mathrm{AA}}^{\mathrm{D}} \lesssim R_{\mathrm{AA}}^{\mathrm{B}}$. The enhancement with respect to unity of heavy-to-light $R_{\mathrm{AA}}$ ratios have been suggested [9] as well-suited observables to test the colour-charge $\left(R_{\mathrm{D} / h}=R_{\mathrm{AA}}^{\mathrm{D}} / R_{\mathrm{AA}}^{h}\right)$ and mass $\left(R_{\mathrm{B} / h}=R_{\mathrm{AA}}^{\mathrm{B}} / R_{\mathrm{AA}}^{h}\right)$ dependence of parton energy loss.

The azimuthal anisotropy of particle production in non-central events is regarded as a powerful tool to study the early stage of the collision. At the LHC, the large cross section for heavy-quark production will allow the direct measurement of the charm and beauty mesons azimuthal anisotropy coefficient $v_{2}$ up to large transverse momenta. Depending on the considered $p_{\mathrm{t}}$ range, the measurement of the $\mathrm{D}$ and $\mathrm{B}$ mesons $v_{2}$ probes: (a) the degree of thermalisation of $\mathrm{c}$ and $\mathrm{b}$ quarks in the expanding medium, at low and intermediate momenta ( $\lesssim 10 \mathrm{GeV} / c$ ), where elliptic flow would be induced by collective pressure effects; (b) the in-medium path-length dependence of heavy-quark energy loss in the almond-shaped partonic system, at higher momenta $(\gtrsim 10 \mathrm{GeV} / c)$.

The measurement of D and B meson production cross sections will also serve as a baseline for the study of medium effects on quarkonia. Two of the most interesting items in the quarkonia sector at the LHC will be: (a) understanding the interplay between suppression and regeneration for $\mathrm{J} / \psi$ production in a medium containing on the order of $100 \mathrm{c} \overline{\mathrm{c}}$ pairs; (b) measuring for the first time medium effects on the bottomonia resonances, expected to be available with sufficient yields at the LHC. On this point, the predicted suppression pattern as a function of the plasma temperature is particularly interesting (see [10] and references therein): the $\Upsilon$ would melt at about $2.5 T_{\mathrm{c}} \approx 420 \mathrm{MeV}$, a temperature that would be reached only at the LHC, while the $\Upsilon^{\prime}$ would melt at the same temperature as the $\mathrm{J} / \psi$, about $1.2 T_{\mathrm{c}}$. It will thus be important for the experiments to be able to measure also the $\Upsilon^{\prime}$, because, at variance with 
the $\mathrm{J} / \psi$, it is expected to have a small regeneration probability and it would be very useful to disentangle $\mathrm{J} / \psi$ suppression and regeneration. We refer to [10] for a detailed review on quarkonia phenomenology. Finally, we note that, in order to study the medium effects on charmonia, it will be mandatory to measure the fraction of secondary charmonia from $\mathrm{B}$ decays, expected to be about $20 \%$ for $\mathrm{J} / \psi$ and $40 \%$ for $\psi^{\prime}$, in absence of medium-induced effects.

\section{ALICE, ATLAS and CMS as heavy-flavour detectors}

Three experiments will participate in the LHC heavy-ion program: ALICE, the dedicated heavy-ion experiment [11,12]; CMS, with a strong heavy-ion program $[13,14]$; ATLAS, which only recently expressed interest in participating $[15,16]$. The three detectors have different features and design requirements, but all of them are expected to have excellent capabilities for heavyflavour measurements. Their complementarity will provide a very broad coverage in terms of phase-space, decay channels and observables.

Experimentally, the two key elements for a rich heavy-flavour program are: tracking/vertexing and particle identification.

Open charm and beauty mesons have typical life-times of few hundred microns ( $c \tau$ values are about $125-300 \mu \mathrm{m}$ for D's and $500 \mu \mathrm{m}$ for B's) and the most direct detection strategy is the identification of single tracks or vertices that are displaced from the interaction vertex. The detector capability to perform this task is determined by the impact parameter ${ }^{1}\left(d_{0}\right)$ resolution. All experiments will be equipped with high position-resolution silicon-detector layers, including pixels, for precise tracking and impact parameter measurement also in the high-multiplicity environment of central $\mathrm{Pb}-\mathrm{Pb}$ collisions. Tracking is done in the central pseudorapidity region: $|\eta|<0.9$ for ALICE and $|\eta| \lesssim 3$ for CMS and ATLAS. In Fig. 1 we show the $d_{0}$ resolution of ALICE, ATLAS and CMS. The $d_{0}$ resolutions are quite similar and better than $50 \mu \mathrm{m}$ for $p_{\mathrm{t}} \gtrsim 1.5-3 \mathrm{GeV} / c$. One of the main differences between the three experiments is given by the magnetic field value, that determines the $p_{\mathrm{t}}$ resolution at high $p_{\mathrm{t}}$ on one hand, and the low $p_{\mathrm{t}}$ reach on the other. ALICE $(0.5 \mathrm{~T})$ has a very low $p_{\mathrm{t}}$ cutoff of $0.2 \mathrm{GeV} / c$ and a $p_{\mathrm{t}}$ resolution of about $5 \%$ at $100 \mathrm{GeV} / c$, while CMS (4 T) and ATLAS (2 T) have a higher cutoff of $1 \mathrm{GeV} / c$ and a resolution of $2 \%$ at $100 \mathrm{GeV} / c$.

Both lepton and hadron identification are important for heavy-flavour detection. $\mathrm{D}$ and $\mathrm{B}$ mesons have relatively-large branching ratios (BR) in the semi-leptonic channels, $\simeq 10 \%$ to electrons and $\simeq 10 \%$ to muons, and inclu-

1 We define as impact parameter the distance of closest approach to the interaction vertex of the track projection in the plane transverse to the beam direction. 


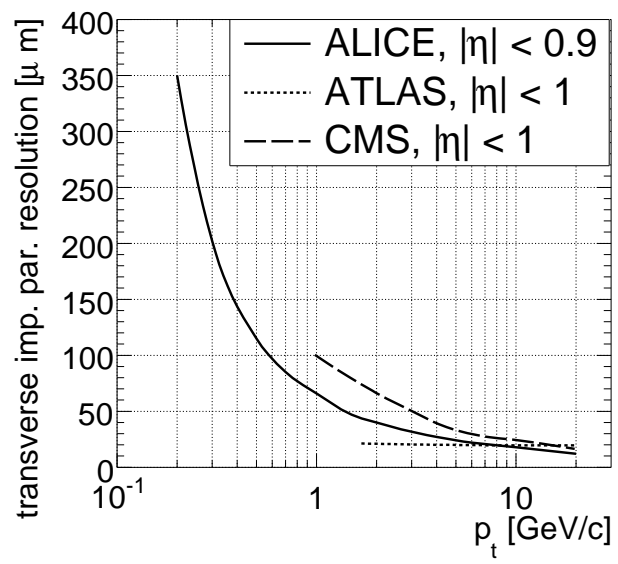

Fig. 1. Transverse track impact parameter resolutions for the ALICE [11], ATLAS [17] and CMS [14] detectors in $\mathrm{Pb}-\mathrm{Pb}$ collisions.

sive cross-section measurements can be performed via single leptons or dileptons. Moreover, quarkonia production is measured using dimuon or dielectron invariant-mass analyses. ALICE can identify electrons with $p_{\mathrm{t}}>1 \mathrm{GeV} / c$ and $|\eta|<0.9$, via transition radiation and $\mathrm{d} E / \mathrm{d} x$ measurements, and muons in the forward region, $-4<\eta<-2.5$, which allows a very low $p_{\mathrm{t}}$ cutoff of $1 \mathrm{GeV} / c$. CMS and ATLAS have a broad pseudorapidity coverage for muons, $|\eta|<2.4$ and $|\eta|<2.7$, respectively, but they have an higher $p_{\mathrm{t}}$ cutoff of $\approx 4 \mathrm{GeV} / c$. Both CMS and ATLAS have high-resolution electromagnetic calorimeters that can be used to identify electrons, although performance studies for heavy-ion collisions have not been carried out yet. Semi-leptonic inclusive measurements do not provide direct information on the $\mathrm{D}(\mathrm{B})$-meson $p_{\mathrm{t}}$ distribution, especially at low $p_{\mathrm{t}}$, because of the weak correlation between the lepton and meson momenta. Therefore, for charm in particular, the reconstruction of exclusive (hadronic) decays is preferable. In this case, in a high-multiplicity environment, hadron identification allows a more effective rejection of the large combinatorial background in the low- $p_{\mathrm{t}}$ region. ALICE disposes of $\pi / \mathrm{K} / \mathrm{p}$ separation via $\mathrm{d} E / \mathrm{d} x$ and time-of-flight measurement for $p<3-4 \mathrm{GeV} / c$ and $|\eta|<0.9$.

\section{Open charm and beauty capabilities}

In this section we present the expected capability of ALICE for the measurement of D and B meson production. Detailed studies for ATLAS and CMS have not been performed yet. However, given the features that we have just discussed, these experiments can be expected to have similar performance as ALICE, though with a more limited low- $p_{\mathrm{t}}$ reach, because of the lack of hadron identification and of the $\approx 4 \mathrm{GeV} / c$ threshold for muons. 

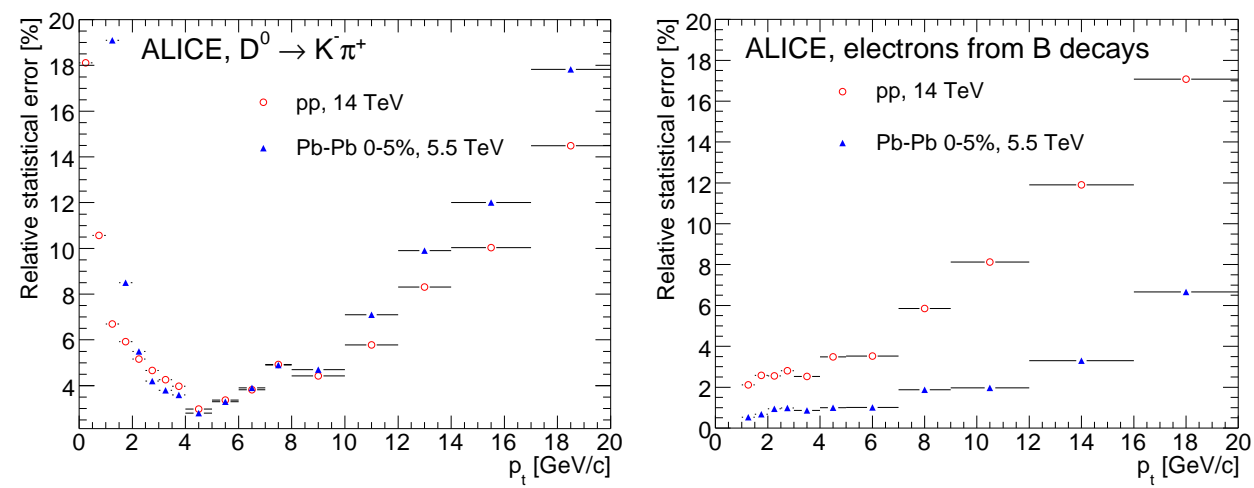

Fig. 2. Expected relative statistical errors for the measurement in ALICE of the production cross sections of $\mathrm{D}^{0}$ (left) and of B-decay electrons (right), in $0-5 \%$ central $\mathrm{Pb}-\mathrm{Pb}$ collisions and in pp collisions.

Exclusive charm reconstruction in ALICE. One of the most promising channels for open charm detection is the $\mathrm{D}^{0} \rightarrow \mathrm{K}^{-} \pi^{+}$decay (and charge conjugate) that has a BR of $3.8 \%$. The main feature of this decay topology is the presence of two tracks with impact parameters $d_{0} \sim 100 \mu \mathrm{m}$. The detection strategy to cope with the large combinatorial background from the underlying event is based on the selection of displaced-vertex topologies, i.e. two tracks with large impact parameters and good alignment between the $\mathrm{D}^{0}$ momentum and flightline, and on invariant-mass analysis to extract the signal yield [12]. As shown in Fig. 2 (left), the accessible $p_{\mathrm{t}}$ range is $1-20 \mathrm{GeV} / c$ for $\mathrm{Pb}-\mathrm{Pb}$ and $0.5-$ $20 \mathrm{GeV} / c$ for pp, with statistical errors better than $15-20 \%$ at high $p_{\mathrm{t}}$. The systematic errors (acceptance and efficiency corrections, centrality selection for $\mathrm{Pb}-\mathrm{Pb}$ ) are expected to be smaller than $20 \%$.

Beauty via single electrons in ALICE. The main sources of background electrons are: decays of $\mathrm{D}$ mesons; $\pi^{0}$ Dalitz decays and decays of light vector mesons (e.g. $\rho$ and $\omega$ ); conversions of photons in the beam pipe or in the inner detector layer and pions misidentified as electrons. Given that electrons from beauty have average impact parameter $d_{0} \simeq 500 \mu \mathrm{m}$ and a hard momentum spectrum, it is possible to obtain a high-purity sample with a strategy that relies on: electron identification with a combined $\mathrm{d} E / \mathrm{d} x$ and transition radiation selection; impact parameter cut to reject misidentified $\pi^{ \pm}$and $\mathrm{e}^{ \pm}$from Dalitz decays and $\gamma$ conversions, and to reduce the charm-decay component. As an example, with $d_{0}>200 \mu \mathrm{m}$ and $p_{\mathrm{t}}>2 \mathrm{GeV} / c$, the expected statistics of electrons from $\mathrm{b}$ decays is $8 \times 10^{4}$ for $10^{7}$ central $\mathrm{Pb}-\mathrm{Pb}$ events, allowing the measurement of electron-level $p_{\mathrm{t}}$-differential cross section in the range $2<p_{\mathrm{t}}<20 \mathrm{GeV} / c$. In Fig. 2 (right) we show the expected statistical errors on the measurement of the cross section of electrons from $b$ decays.

Beauty via muons in ALICE. B production in $\mathrm{Pb}-\mathrm{Pb}$ collisions can be measured also in the ALICE muon spectrometer $(-4<\eta<-2.5)$ analyzing the single-muon $p_{\mathrm{t}}$ distribution [12]. The main backgrounds to the 'beauty muon' 

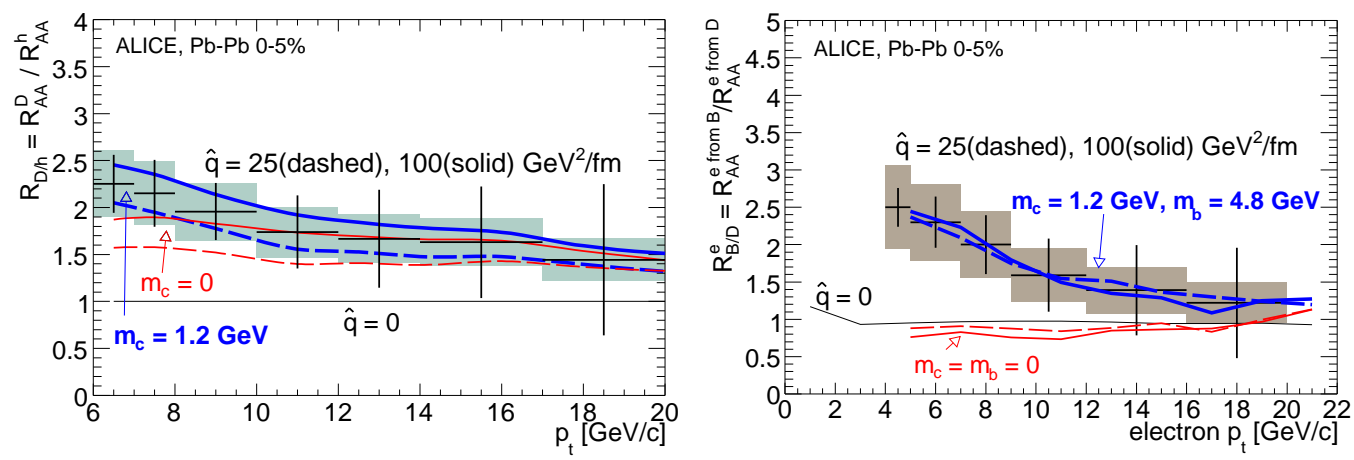

Fig. 3. Ratio of the nuclear modification factors for $\mathrm{D}^{0}$ mesons and for charged hadrons (left) and ratio of the nuclear modification factors for B-decay and for D-decay electrons (right). Errors corresponding to the centre of the prediction bands for massive quarks are shown: bars = statistical, shaded area = systematic.

signal are $\pi^{ \pm}, \mathrm{K}^{ \pm}$and charm decays. The cut $p_{\mathrm{t}}>1.5 \mathrm{GeV} / c$ is applied to all reconstructed muons in order to increase the signal-to-background ratio. Then, a fit technique allows to extract a $p_{\mathrm{t}}$ distribution of muons from B decays. Since only minimal cuts are applied, the statistical errors are expected to be smaller than $5 \%$ up to muon $p_{\mathrm{t}} \approx 30 \mathrm{GeV} / c$.

Heavy-to-light ratios in ALICE. ALICE investigated the possibility of using the described charm and beauty measurements to study the dependences of parton energy loss. The expected experimental errors on these observables are compared to recent theoretical predictions from parton energy loss [9]. The sensitivity to the heavy-to-light ratios $R_{\mathrm{D} / h}=R_{\mathrm{AA}}^{\mathrm{D}} / R_{\mathrm{AA}}^{h}$ and $R_{\mathrm{B} / \mathrm{D}}=$

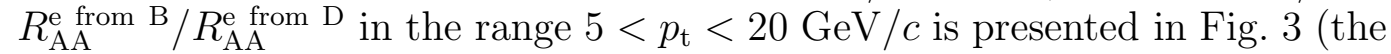
$p_{\mathrm{t}}$ distribution of D-decay electrons will be calculated from the measured $\mathrm{D}^{0}$ $p_{\mathrm{t}}$ distribution). Predictions with and without the effect of the heavy-quark mass, for a medium transport coefficient in the range $25-100 \mathrm{GeV}^{2} / \mathrm{fm}$, are shown. For $5<p_{\mathrm{t}}<10 \mathrm{GeV} / c$, the measurement of the expected enhancement of heavy-to-light ratios with respect to unity appears to be feasible.

\section{Quarkonia capabilities}

Figure 4 shows the schematic acceptances for charmonia and bottomonia in the $\left(y, p_{\mathrm{t}}\right)$ plane. ALICE can detect quarkonia in the dielectron channel at central rapidity $(|y| \lesssim 1)$ and in the dimuon channel at forward rapidity $(-4<y<-2.5)$. In both channels the acceptance extends down to zero transverse momentum, since the minimum $p_{\mathrm{t}}$ is $1 \mathrm{GeV} / c$ for both electrons and muons. ATLAS and CMS will use only dimuons and they have similar acceptances, covering $p_{\mathrm{t}} \gtrsim 3 \mathrm{GeV} / c$ and $|y| \lesssim 2.5$. CMS studies indicate that, near the edges of the pseudorapidity window, there is some acceptance down to $p_{\mathrm{t}} \approx 1.5 \mathrm{GeV} / c$. We emphasized the importance of separating the $\Upsilon$ and $\Upsilon^{\prime}$; 

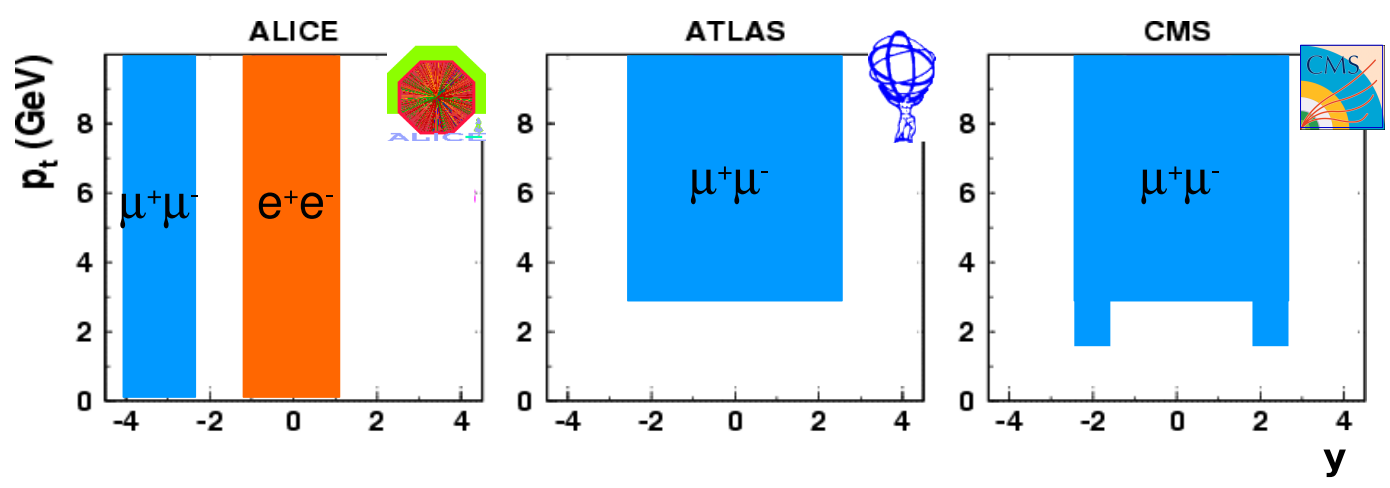

Fig. 4. Schematic $\left(y, p_{\mathrm{t}}\right)$ quarkonia acceptances for ALICE, ATLAS and CMS.

given that the mass difference between the two bottomonia is about $500 \mathrm{MeV}$, a mass resolution of order $100 \mathrm{MeV}$ at $M_{\ell^{+} \ell^{-}} \sim 10 \mathrm{GeV}$, i.e. $\sigma_{M} / M \approx 1 \%$, is required. CMS and ALICE fulfill this requirement, while for ATLAS the $\Upsilon / \Upsilon^{\prime}$ separation will be more difficult. We report in Table 2 a summary of the expected quarkonia performances, including the mass resolution, the list of measurable states and the $p_{\mathrm{t}}$ coverage for $\mathrm{J} / \psi$ and $\Upsilon$. For illustration, in Fig. 5 we show the simulated dilepton mass spectra in the $\Upsilon$ region after background subtraction $[12,16,14]$. All three experiments will be able to measure the fraction of $\mathrm{J} / \psi$ that feed-down from $\mathrm{B}$ decays, by studying the impact parameter distribution of the dilepton pairs.

Table 2

Expected quarkonia performances in central $\mathrm{Pb}-\mathrm{Pb}$ for ALICE, ATLAS and CMS.

\begin{tabular}{|c|c|c|c|c|c|}
\hline & & $\mathrm{ALICE}^{+} \mathrm{e}^{-}$ & $\operatorname{ALICE} \mu^{+} \mu^{-}$ & ATLAS $\mu^{+} \mu^{-}$ & $\operatorname{CMS} \mu^{+} \mu^{-}$ \\
\hline \multirow[t]{4}{*}{$\psi$} & $y$ acc. & $|y|<1$ & $-4<y<-2.5$ & $|y|<2.5$ & $|y|<2$ \\
\hline & Mass res. & $35 \mathrm{MeV}$ & $65 \mathrm{MeV}$ & $70 \mathrm{MeV}$ & $37 \mathrm{MeV}$ \\
\hline & Meas. states & $\psi \mathrm{OK} ; \psi^{\prime} ?$ & $\psi \mathrm{OK} ; \psi^{\prime} ?$ & $\psi \mathrm{OK} ; \psi^{\prime} ?$ & $\psi \mathrm{OK} ; \psi^{\prime} ?$ \\
\hline & $p_{\mathrm{t}}$ range & $0-10 \mathrm{GeV} / c$ & $0-20 \mathrm{GeV} / c$ & not studied & few-20 GeV/c \\
\hline \multirow[t]{3}{*}{$\Upsilon$} & $y$ acc. & $|y|<1$ & $-4<y<-2.5$ & $|y|<2.5$ & $|y|<0.8(2.4)$ \\
\hline & Mass res. & $90 \mathrm{MeV}$ & $90 \mathrm{MeV}$ & $145 \mathrm{MeV}$ & $54(85) \mathrm{MeV}$ \\
\hline & $\begin{array}{l}\text { Meas. states } \\
p_{\mathrm{t}} \text { range }\end{array}$ & $\begin{array}{l}\Upsilon \mathrm{OK} ; \Upsilon^{\prime} ? \\
\text { not studied }\end{array}$ & $\begin{array}{c}\Upsilon, \Upsilon^{\prime} \mathrm{OK} ; \Upsilon^{\prime \prime} ? \\
0-8 \mathrm{GeV} / c\end{array}$ & $\begin{array}{c}\Upsilon, \Upsilon^{\prime} \mathrm{OK} ; \Upsilon^{\prime \prime} ? \\
\text { not studied }\end{array}$ & $\begin{array}{l}\Upsilon, \Upsilon^{\prime} \mathrm{OK} ; \Upsilon^{\prime \prime} ? \\
\text { few-10 GeV/c }\end{array}$ \\
\hline
\end{tabular}
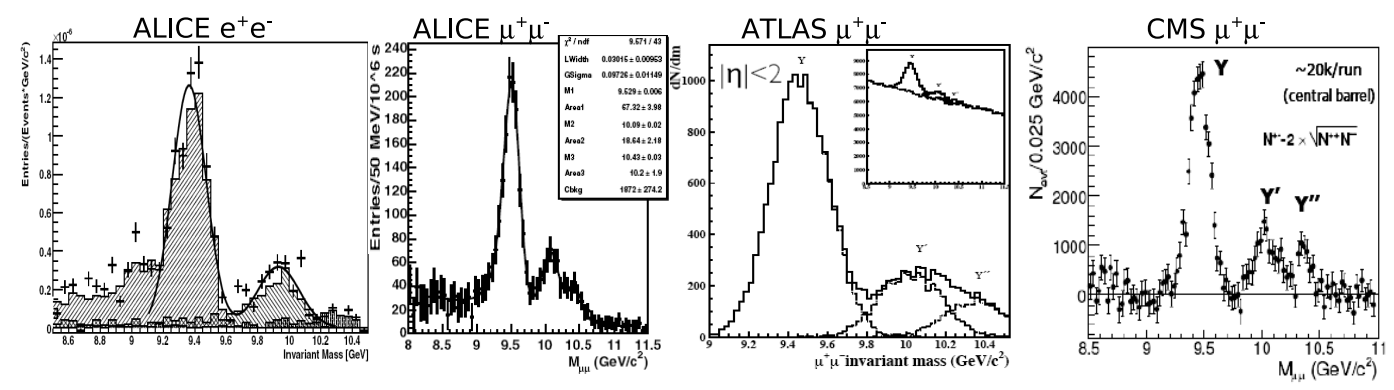

Fig. 5. The $\Upsilon$ family in central $\mathrm{Pb}-\mathrm{Pb}$ collisions, as pictured by ALICE [12], ATLAS [16] and CMS [14] in one month of data-taking. 


\section{Summary}

We have discussed how heavy quarks, abundantly produced at LHC energies, will allow to address several physics issues in heavy-ion collisions. In particular, they provide tools to probe the density, via parton energy loss and its predicted mass dependence, and temperature, via quarkonia successive dissociation patterns, of the high-density $\mathrm{QCD}$ medium formed in $\mathrm{Pb}-\mathrm{Pb}$ collisions. The excellent tracking, vertexing and particle identification performance of ALICE, ATLAS and CMS will allow to fully explore this rich phenomenology.

Acknowledgment. The author is grateful to F. Antinori, P. Crochet, D. d'Enterria, G. Martinez, A. Morsch, G. Roland, J. Schukraft, H .Takai and B. Wyslouch for valuable help in the compilation of the experiments performance results.

\section{References}

[1] F. Karsch, these proceedings.

[2] C.A. Salgado, these proceedings; M. Djordjevic, these proceedings.

[3] N. Carrer and A. Dainese, ALICE-INT-2003-019 (2003) hep-ph/0311225.

[4] M. Mangano, P. Nason and G. Ridolfi, Nucl. Phys. B373 (1992) 295.

[5] K.J. Eskola et al., Eur. Phys. J. C9 (1999) 61.

[6] A. Accardi et al, hep-ph/0308248, in CERN Yellow Report 2004-009.

[7] Yu.L. Dokshitzer and D.E. Kharzeev, Phys. Lett. B519 (2001) 199.

[8] N. Armesto et al., Phys. Rev. D69 (2004) 114003.

[9] N. Armesto et al., Phys. Rev. D71 (2005) 054027.

[10] H. Satz, these proceedings.

[11] ALICE Coll., Physics Performance Report Vol. I, J. Phys. G30 (2004) 1517.

[12] ALICE Coll., Physics Performance Report Vol. II, J. Phys. G32 (2006) 1295.

[13] CMS Coll., Heavy-Ion Group http://cmsdoc.cern.ch/cms/PRS/hi/

[14] G. Roland, these proceedings.

[15] ATLAS Coll., Heavy-ion Letter of Intent, CERN/LHCC 2004-009 (2004).

[16] H. Takai, these proceedings.

[17] L. Rosselet, talk at "International workshop on heavy flavor production in heavy ion collisions at the LHC", ECT*, Trento, 6-10 September 2006. 